\title{
Grid-Connection Half-Bridge PV Inverter System for Power Flow Controlling and Active Power Filtering
}

\author{
Chih-Lung Shen and Jye-Chau Su \\ Department of Electronic Engineering, National Kaohsiung First University of Science and Technology, Nanzih, 1, University Road, \\ Yuanchau, Kaohsiung 824, Taiwan \\ Correspondence should be addressed to Chih-Lung Shen, clshen@ccms.nkfust.edu.tw
}

Received 19 March 2012; Accepted 11 May 2012

Academic Editor: G. N. Tiwari

Copyright (๑) 2012 C.-L. Shen and J.-C. Su. This is an open access article distributed under the Creative Commons Attribution License, which permits unrestricted use, distribution, and reproduction in any medium, provided the original work is properly cited.

\begin{abstract}
A half-bridge photovoltaic (PV) system is proposed, which can not only deal with bidirectional power flowing but also improve power quality. According to varying insolation, the system conditions real power for $\mathrm{dc}$ and ac loads to accommodate different amounts of PV power. Furthermore, the system eliminates current harmonics and improves power factor simultaneously. As compared with conventional PV inverter, the total number of active switches and current sensors can be reduced so that its cost is lower significantly. For current command determination, a linear-approximation method (LAM) is applied to avoid the complicated calculation and achieve the maximum power point tracking (MPPT) feature. For current controlling, a directsource-current-shaping (DSCS) algorithm is presented to shape the waveform of line current. Simulation results and practical measurements also demonstrate the feasibility of the proposed half-bridge PV system.
\end{abstract}

\section{Introduction}

Solar energy is clean, pollution-free, and inexhaustible, so developing solar energy power system can solve the energy crisis of exhausting in fossil fuel. Recently, photovoltaic arrays are widely used for power supply [1-14]. PV systems can be briefly classified into stand-alone and grid-connection types. Owing to more flexibility in power conditioning, the study on the grid-connection type stimulates many interests. Figure 1 shows the configuration of a conventional gridconnection PV system, which consists of multiple stages, leading to low efficiency, large volume, and high cost. To improve part of the disadvantages, some researchers have designed two-stage configurations, as shown in Figure 2. For further efficiency improvement and cost reduction, singlestage PV system has been developed [15-18], of which block diagram is shown in Figure 3. Even though the structure of a single-stage PV system is simpler than that of a twostage one, a couple of active switches, current sensors, and corresponding drivers are still needed in the power stage.

In this paper a half-bridge single-stage PV system is proposed to reduce the total number of active switches and current sensors. As a result, the proposed PV inverter system is compact and cost can be reduced significantly. Furthermore, the proposed system can not only process real power bidirectionally but also improve power factor and eliminate harmonic currents. To draw maximum power from PV arrays, a linear-approximation method (LAM) is developed to complete the maximum power point tracking (MPPT). Based on the LAM, a reference dc-link voltage is chosen. With an outer-voltage controller, source current commands are determined, which avoids optimal current determination from complicated calculations. A direct-source-currentshaping (DSCS) algorithm is applied to perform wave shaping for bidirection power flow controlling and power factor improvement. A prototype is established, simulated, tested, and measured. The simulation results and experimental measurements have verified the feasibility of the proposed PV system.

\section{Configuration of the PV System}

Figure 4 illustrates the configuration of the PV power system, which consists of a dc-bus filter, a half-bridge inverter, 


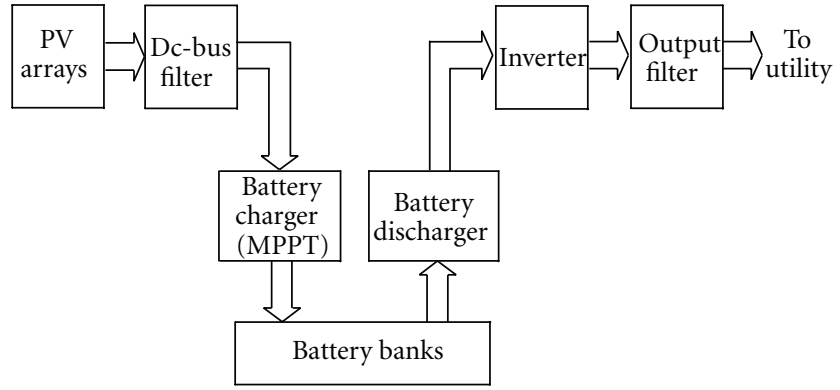

Figure 1: A block diagram of a conventional grid-connection PV system.

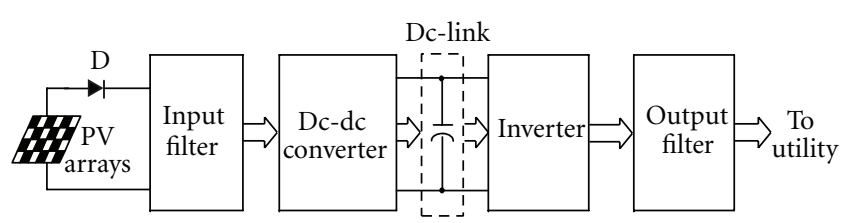

Figure 2: Illustration for a two-stage grid-connection PV system.

an output filter, and a system controller. The half-bridge inverter, which contains two active switches and two dcvoltage-divided capacitors, can process real power bidirectionally. That is, the inverter either transfers PV power to ac side or draws power from utility for dc loads. In addition, the inverter performs current harmonics eliminating and power factor correcting to improve power quality. The dcbus filter suppresses dc-link voltage fluctuations and filters out ac components on the dc side for accurate MPPT, while the output filter serves as an interface between the inverter and the utility to prevent inrush current from occurring. According to dc-link, reference, and line voltages, the system controller implemented in a DSP chip calculates current commands and then determines appropriate switch signals to perform wave shaping. A conceptual block diagram of the system controller is shown in Figure 5.

\section{Operation Principle of the PV System}

To understand the operation principle of the PV system, power flow controlling is discussed. The PV system processes real power, reactive power, and distortion power simultaneously. Figure 6 is the power tetrahedron diagram, which shows the relationship among these types of power. In Figure 6, the $S$ stands for apparent power and is expressed as

$$
S=\sqrt{(\bar{p})^{2}+(\bar{q})^{2}+\left(h_{p w}\right)^{2}}
$$

where $\bar{p}, \bar{q}$, and $h_{p w}$ denote real power, reactive power, and distortion power, respectively. According to different insolation, the PV system can deal with power bidirectionally. Based on $\bar{p}-\bar{q}-h_{p w}$ coordinate frame, Figure 7 shows a trajectory to indicate operation points varying with insolation. From point $a$ to point $b$, during the interval of high insolation the PV system generates solar power to

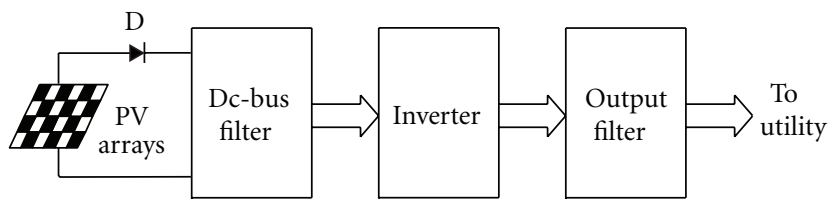

Figure 3: A block diagram to represent a single-stage gridconnection PV system.

supply dc loads and ac loads and inject real power into utility. In addition, the half-bridge inverter processes reactive power and distortion power for ac loads so as to improve power factor. A corresponding power flow is illustrated in Figure 8. From point $b$ to point $c$, during the interval of medium insolation the system supplies power for dc loads and part of real power for ac loads and the insufficient draws from utility. Figure 9 is the related power flow. From point $c$ to point $d$, during the interval of low insolation the PV arrays cannot feed total amount of dc demanded power so that the inverter transforms ac power to $\mathrm{dc}$ one for $\mathrm{dc}$ loads and deals with reactive power and distortion power for ac loads simultaneously. The corresponding power flow is shown in Figure 10. At point $d$, during the interval of no insolation the inverter processes real power for dc loads and deals with reactive power and distortion power for ac loads. Figure 11 shows the power flow direction.

\section{Derivation of Current Commands}

In the PV system, once a current command is determined, the output current of the half-bridge inverter will trace the waveform of the reference current to perform power flow controlling and power quality improvement. In the following, an optimal current command is derived.

According to the current and voltage definitions shown in Figure 4 , the line voltage $v_{s}(t)$ and nonlinear load current $i_{L}(t)$ are expressed as

$$
\begin{gathered}
v_{s}(t)=\sqrt{2} V_{\mathrm{rms}} \sin (\omega t-\phi), \\
i_{L}(t)=\sum_{n=1}^{\infty} \sqrt{2} I_{n} \sin \left(n \omega t-\theta_{n}\right),
\end{gathered}
$$

respectively. Then, the load instantaneous real power $\left(p_{L}(t)\right)$ and instantaneous reactive power $\left(q_{L}(t)\right)$ can be calculated as follows:

$$
\begin{aligned}
p_{L}(t)= & v_{s}(t) i_{L}(t) \\
= & V_{\mathrm{rms}} I_{1} \cos \left(\phi-\theta_{1}\right)-V_{\mathrm{rms}} I_{1} \cos \left(2 \omega t+\phi+\theta_{1}\right) \\
& +\sum_{n=2}^{\infty} 2 V_{\mathrm{rms}} I_{n} \sin \left(n \omega t+\theta_{n}\right) \sin (\omega t+\phi) \\
= & \bar{p}_{L}+\tilde{p}_{L},
\end{aligned}
$$




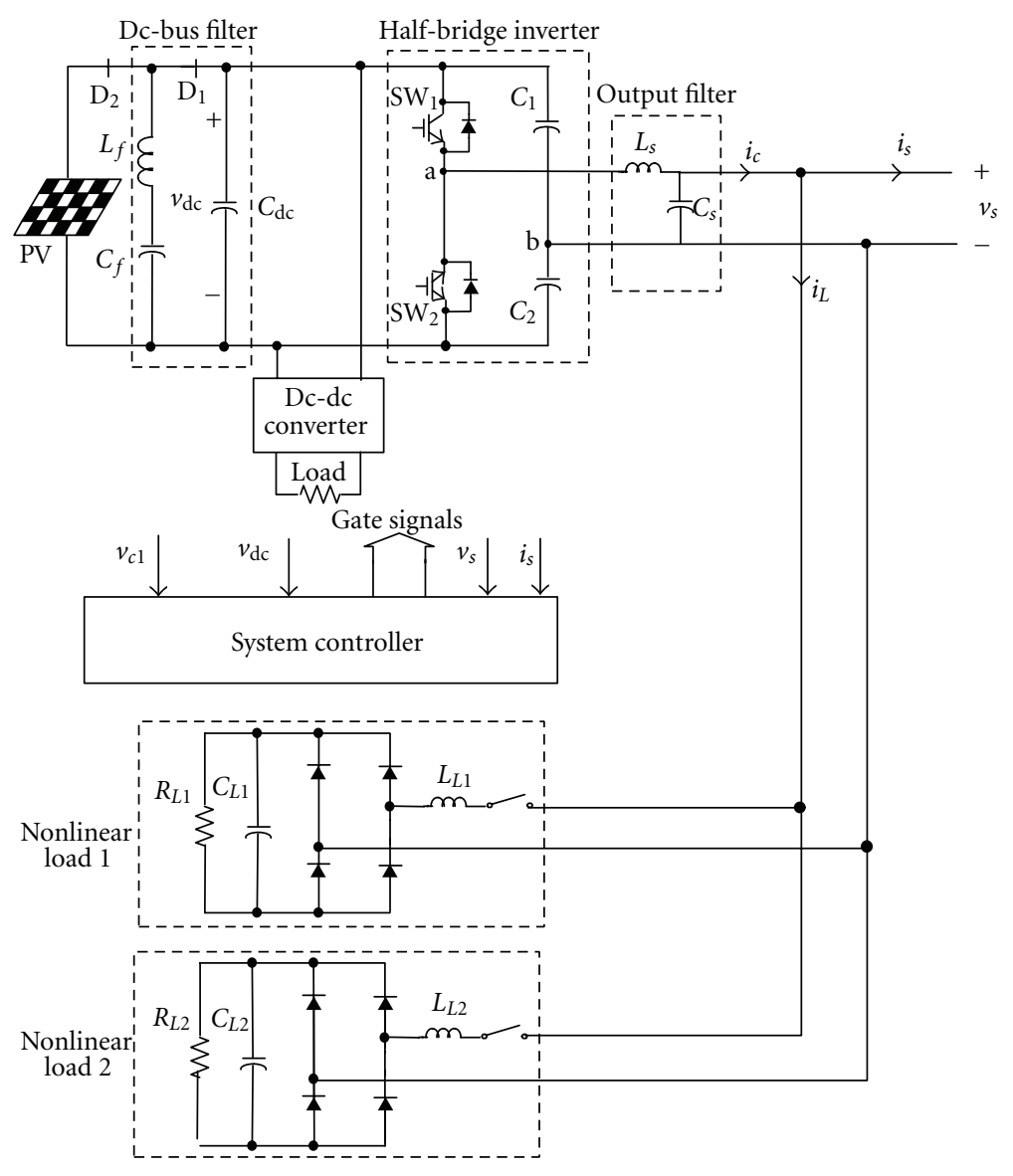

FIgURE 4: Configuration of the PV inverter system.

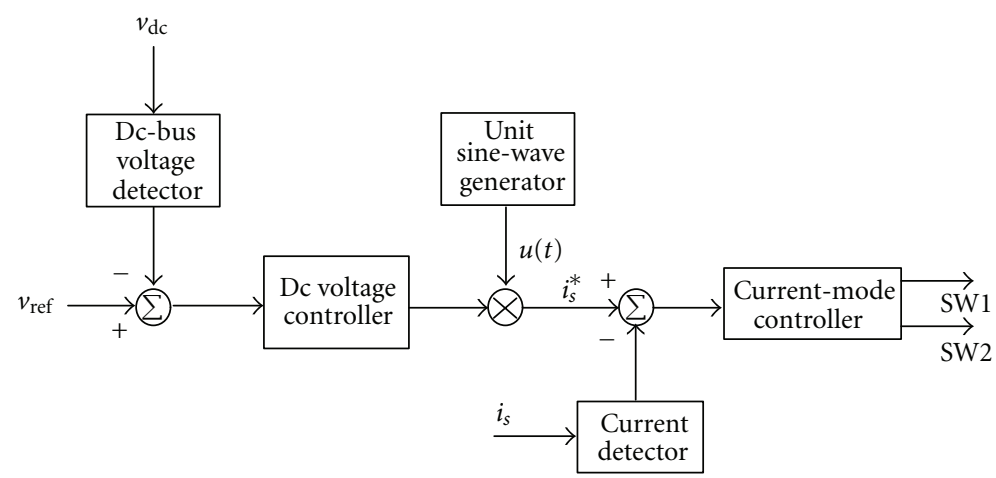

Figure 5: A block diagram of the system controller.

where

$$
\begin{aligned}
\bar{P}_{L}= & V_{\mathrm{rms}} I_{1} \cos \left(\phi-\theta_{1}\right), \\
\tilde{p}_{L}= & V_{\mathrm{rms}} I_{1} \cos \left(2 \omega t+\phi+\theta_{1}\right) \\
& +\sum_{n=2}^{\infty} 2 V_{\mathrm{rms}} I_{n} \sin \left(n \omega t+\theta_{n}\right) \sin (\omega t+\phi) .
\end{aligned}
$$

Notation $\bar{p}_{L}$ represents the constant part and $\tilde{p}_{L}$ denotes the variant component. The instantaneous reactive power can be obtained by multiplying the nonlinear load current with a $90^{\circ}$-shifted voltage as follows:

$$
\begin{aligned}
q_{L}(t)= & v_{s}^{\prime}(t) i_{L}(t) \\
= & V_{\mathrm{rms}} I_{1} \sin \left(\phi-\theta_{1}\right)-V_{\mathrm{rms}} I_{1} \sin \left(2 \omega t+\phi+\theta_{1}\right) \\
& -\sum_{n=2}^{\infty} 2 V_{\mathrm{rms}} I_{n} \sin \left(n \omega t+\theta_{n}\right) \cos (\omega t+\phi) \\
= & \bar{q}_{L}+\tilde{q}_{L},
\end{aligned}
$$




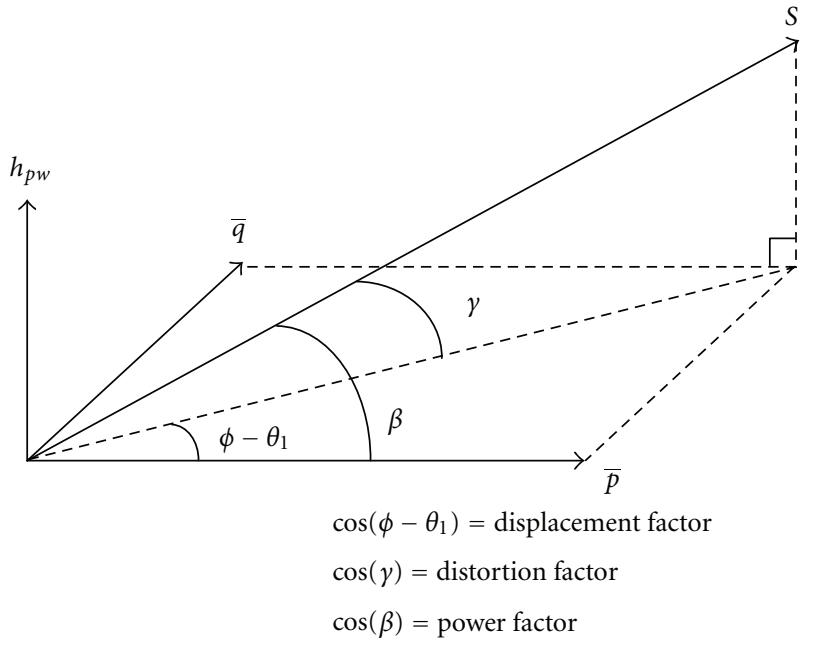

Figure 6: Power tetrahedron diagram.

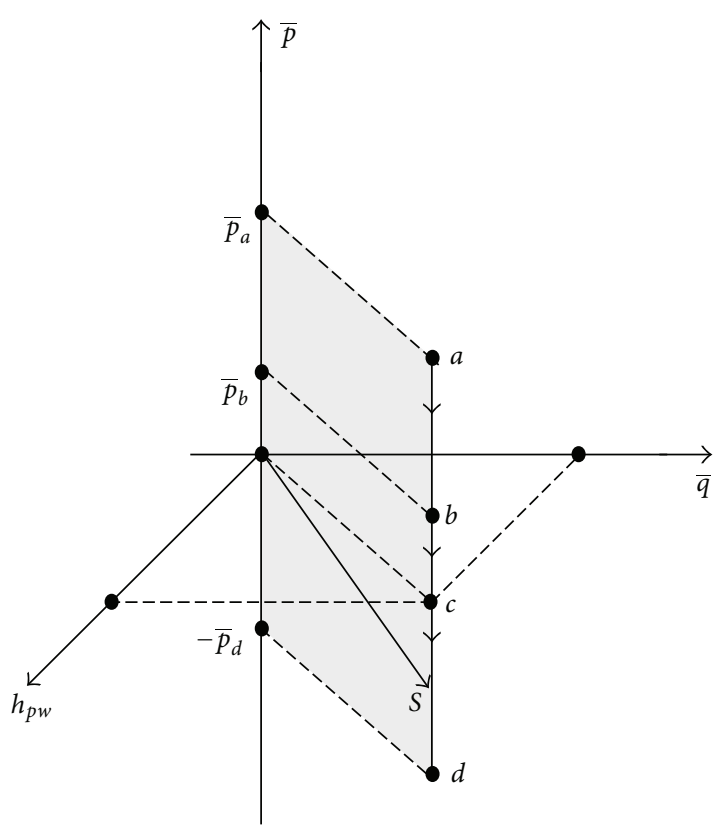

FIGURE 7: A trajectory to indicate operation points varying with insolation.

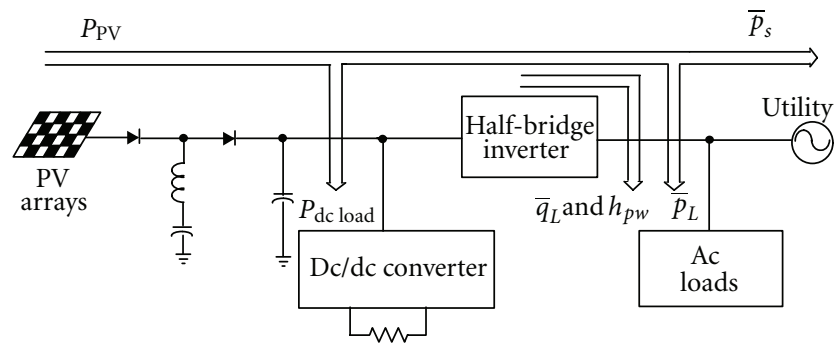

FIGURE 8: Illustration of power flow during the interval of high insolation.

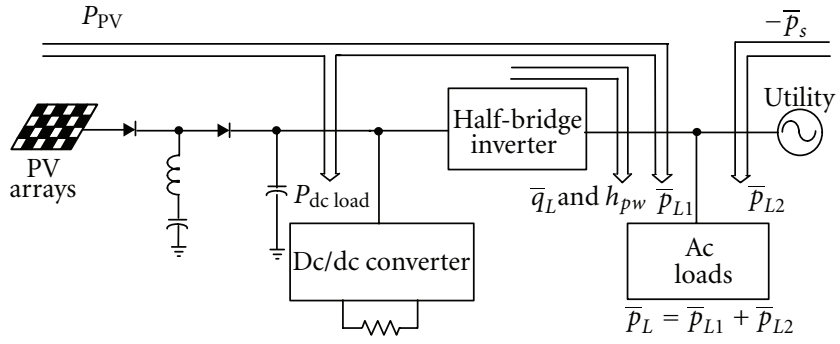

FIGURE 9: Illustration of power flow during the interval of medium insolation.

where $v_{s}^{\prime}(t)$ is the line voltage shifted by $90^{\circ}, \tilde{q}_{L}$ is the constant part, and $\tilde{q}_{L}$ is the variant component of instantaneous reactive power. Apparent power is determined by

$$
\begin{aligned}
S & =V_{\mathrm{rms}} \sqrt{\sum_{n=1}^{\infty} I_{n}^{2}} \\
& =\sqrt{\left[V_{\mathrm{rms}} I_{1} \cos \left(\phi-\theta_{1}\right)\right]^{2}+\left[V_{\mathrm{rms}} I_{1} \sin \left(\phi-\theta_{1}\right)\right]^{2}+\sum_{n=2}^{\infty} V_{\mathrm{rms}}^{2} I_{n}^{2},}
\end{aligned}
$$

in which the first, second, and third terms are the square of real, reactive, and distortion powers, respectively. The reactive and distortion powers of a nonlinear load will be supplied by the PV system. As a result, a compensated line current, of which amplitude depends on PV power, is purely sinusoidal and in phase with line voltage. It can be determined by

$$
i_{s}^{*}=\frac{\sqrt{2}\left(p_{\mathrm{MPPT}}-\bar{p}_{L}(t)\right)}{V_{\mathrm{rms}}} \sin (\omega t-\phi) .
$$

In addition, a corresponding inverter output current is expressed as

$$
i_{c}^{*}=\frac{\sqrt{2}\left(p_{\mathrm{MPPT}}-\bar{p}_{L}(t)\right)}{V_{\mathrm{rms}}} \sin (\omega t-\phi)+i_{L},
$$

where $p_{\text {MPPT }}$ is the maximum power drawn from the PV arrays and can be represented as

$$
p_{\mathrm{MPPT}}=\left(\nu_{\mathrm{PV}}(t) \cdot i_{\mathrm{PV}}(t)\right)_{\max } .
$$

In (7) and (8), the difference between $p_{\text {MPPT }}$ and $\bar{p}_{L}(t)$ decides the amplitudes of current commands, which can be also obtained from the comparison of the dc-link voltage with a reference voltage. The linear-approximation method (LAM) to achieve the maximum power point tracking (MPPT) is illustrated in Figures 12 and 13, from which it can be found that the trajectories of maximum power point varying with irradiation and temperature are linear. Once the reference voltage corresponding to a maximum power point is determined, maximum power drawing from PV arrays can be readily achieved. In Figure 12, maximum power of the PV arrays is proportional to output voltage with the increasing 


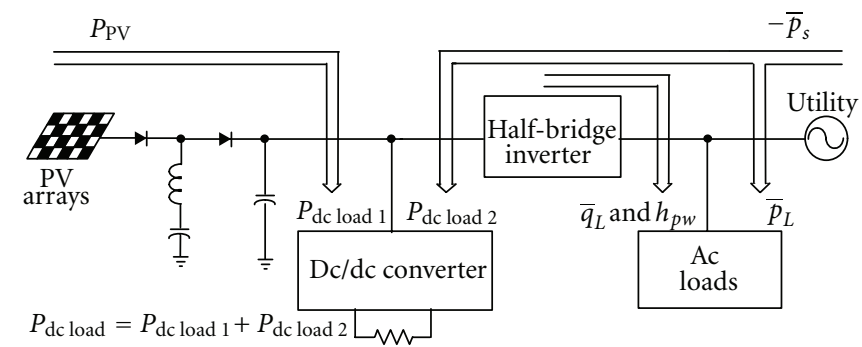

FIGURE 10: Illustration of power flow during the interval of low insolation.

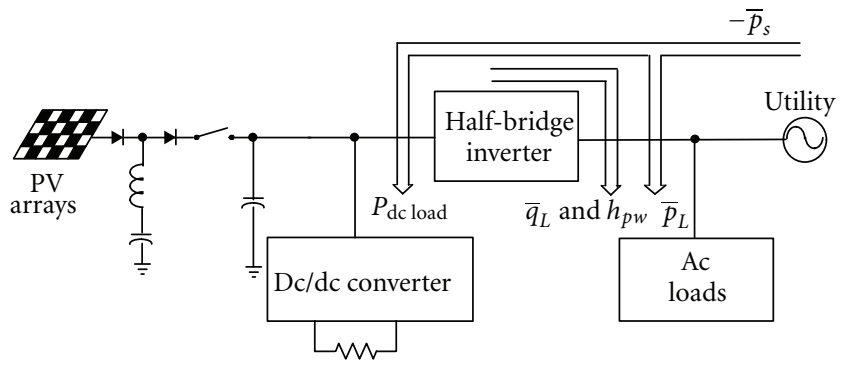

FIGURE 11: Illustration of power flow during the interval of no insolation.

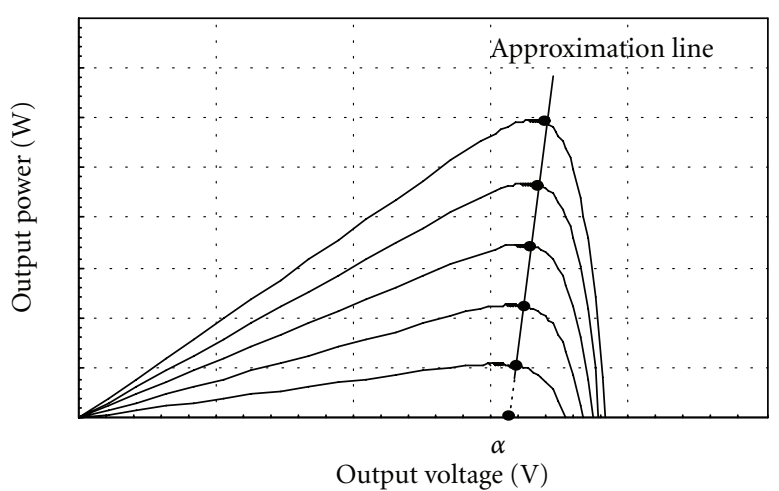

FIGURE 12: Illustration of the trajectory of maximum power point varying with insolation while PV temperature is invariant.

of insolation, while temperature is constant. In this paper, insolation is detected by a photodiode converting luminance into current and then measured by a resistor. According to Figure 12, the MPPT voltage $v_{\text {ref }}^{\prime}$ is determined as

$$
v_{\mathrm{ref}}^{\prime}=\frac{k}{m} i_{p}+\alpha
$$

where $m$ represents the slope of the approximation line, $\alpha$ stands for the crossover point with output-voltage axis, $i_{p}$ is the output current of a photodiode, and $k$ is a coefficient determined by the ratio of luminance to photodiode current. In Figure 13, a maximum power point also varies with temperature such that $v_{\text {ref }}^{\prime}$ should be modified. As illustrated in Figure 14, a maximum power voltage drops linearly with

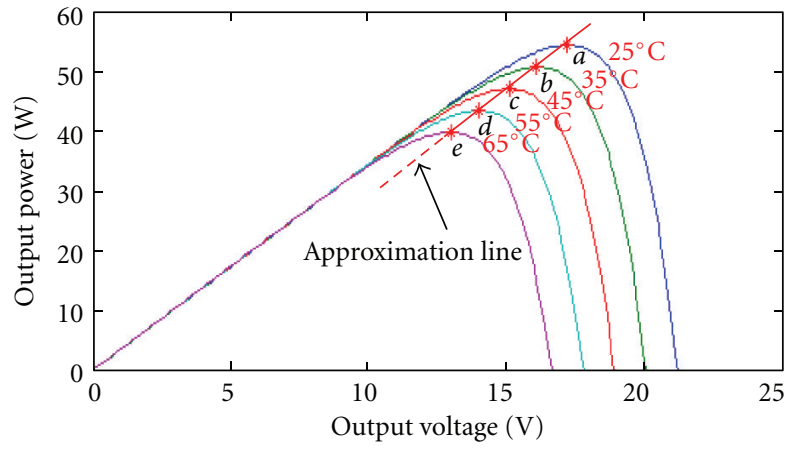

FIGURE 13: Illustration of the trajectory of maximum power point varying with temperature while insolation is constant.

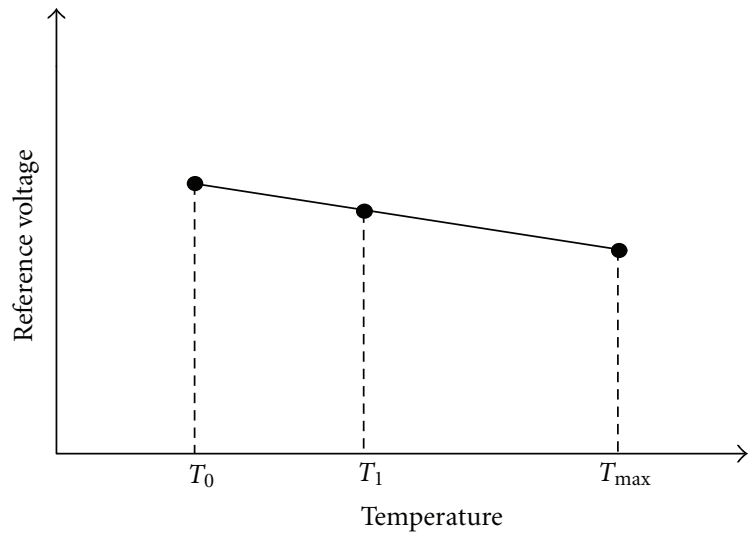

FIGURE 14: Illustration of the relationship between reference voltage and temperature. 


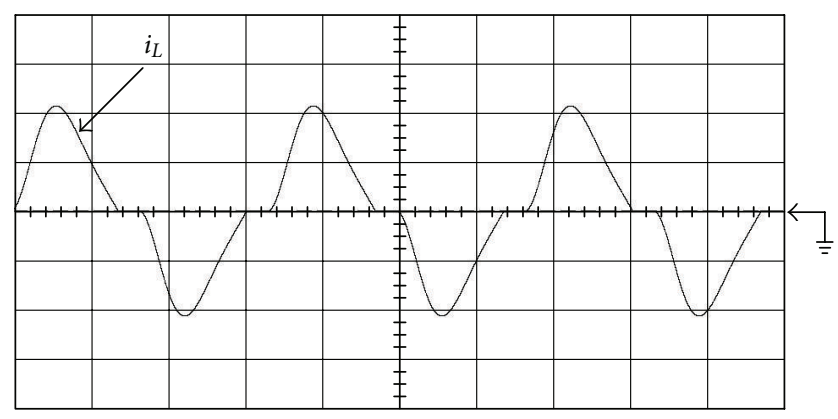

( $i_{L}: 5 \mathrm{~A} / \mathrm{div}$, time: $\left.5 \mathrm{~ms} / \mathrm{div}\right)$

FIGURE 15: Load current while nonlinear loads are connected to utility.

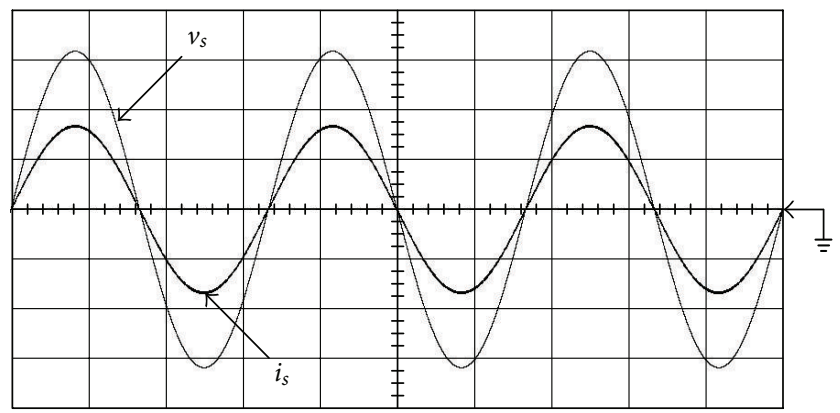

$\left(v_{s}: 50 \mathrm{~V} / \mathrm{div}, i_{s}: 5 \mathrm{~A} / \mathrm{div}\right.$, time: $\left.5 \mathrm{~ms} / \mathrm{div}\right)$

FIGURE 16: Filtered line current and line voltage during the interval of high insolation.

the increasing of temperature under constant insolation. As a result, a reference voltage $v_{\text {ref }}$ can be found by

$$
v_{\text {ref }}=\varsigma v_{\text {ref }}^{\prime}+\beta
$$

where $\zeta$ is temperature compensation coefficient and notation $\beta$ is a constant value.

\section{Simulated and Experimental Results}

An example of $110 \mathrm{~V} 60 \mathrm{~Hz}$ half-bridge PV system is designed, simulated, and implemented, of which operation range of input voltage is from 395 to $420 \mathrm{~V}$. Component values and important parameters are determined as

power switches: IGBT, TOSHIBA GT25Q101, 1200 $\mathrm{V} / 25 \mathrm{~A}$,

PV arrays: SHARP NT-KR5EX (12 pieces in series),

$f_{s}=20 \mathrm{kHz}$,

$C_{1}=C_{2}=C_{\mathrm{dc}}=940 \mu \mathrm{F}, C_{f}=880 \mu \mathrm{F}$,

$L_{s}=4 \mathrm{mH}, L_{f}=2 \mathrm{mH}$,

$v_{\text {ref: }}$ from $395 \mathrm{~V}$ to $420 \mathrm{~V}$,

PV power: from $200 \mathrm{~W}$ to $1.8 \mathrm{~kW}$.

Nonlinear loads are connected to utility, of which power dissipation is $650 \mathrm{~W}$. Figure 15 shows the waveform of

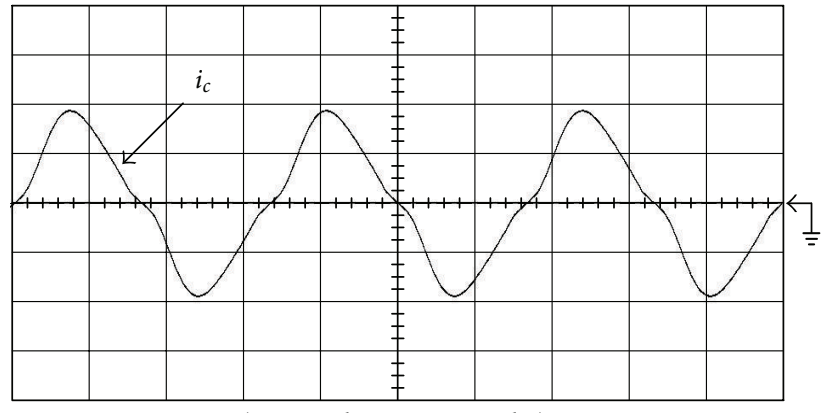

$\left(i_{c}: 10 \mathrm{~A} / \mathrm{div}\right.$, time: $\left.5 \mathrm{~ms} / \mathrm{div}\right)$

FIGURE 17: The corresponding inverter current during the interval of high insolation.

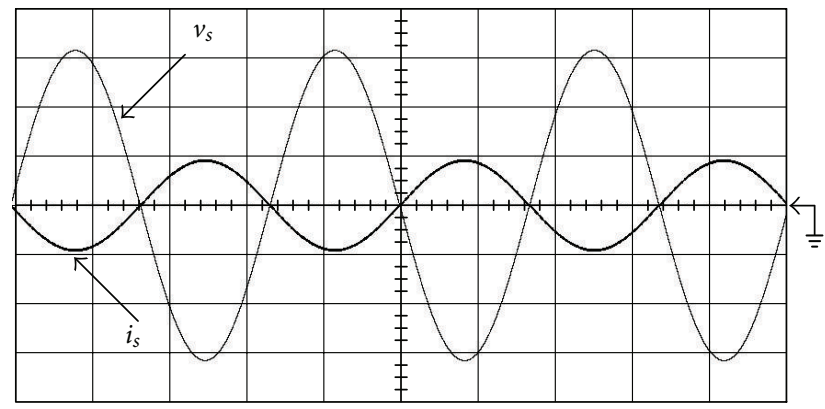

$\left(v_{s}: 50 \mathrm{~A} / \mathrm{div}, i_{s}: 5 \mathrm{~A} / \mathrm{div}\right.$, time: $\left.5 \mathrm{~ms} / \mathrm{div}\right)$

FIGURE 18: Filtered line current and line voltage during the interval of medium insolation.

the load current. During the interval of high insolation, PV arrays generate $1.8 \mathrm{~kW}$. In addition, a reference dclink voltage $v_{\text {ref }}$ is $420 \mathrm{~V}$ based on the LAM for MPPT. The simulated line current and the corresponding inverter current are shown in Figures 16 and 17, respectively. In this period, PV system supplies total amount of demanded power for $\mathrm{dc}$ and ac loads and injects real power into utility. Simultaneously, PV system compensates reactive power and distortion power for nonlinear loads to improve power factor. From Figure 16, it can be observed that line current is sinusoidal and in phase with line voltage. That is, high power factor is achieved and PV power can be injected into utility. During the interval of medium insolation, output power of the PV arrays is $800 \mathrm{~W}$ and reference voltage $v_{\text {ref }}$ is $404 \mathrm{~V}$. PV system provides total amount of power for dc load and part of real power for nonlinear loads. The simulated line current is shown in Figure 18, while Figure 19 is the corresponding inverter current. From Figure 18, it can be found that the line current is purely sinusoidal and $180^{\circ}$ out of phase to line voltage. That is, insufficient power for ac load is fed from utility and power factor correction is performed by the half-bridge inverter simultaneously. During the interval of low insolation, PV power is $200 \mathrm{~W}$ and a reference dc-link voltage is $395 \mathrm{~V}$. Figure 20 shows the simulated line current, and Figure 21 is the corresponding inverter current. Once 


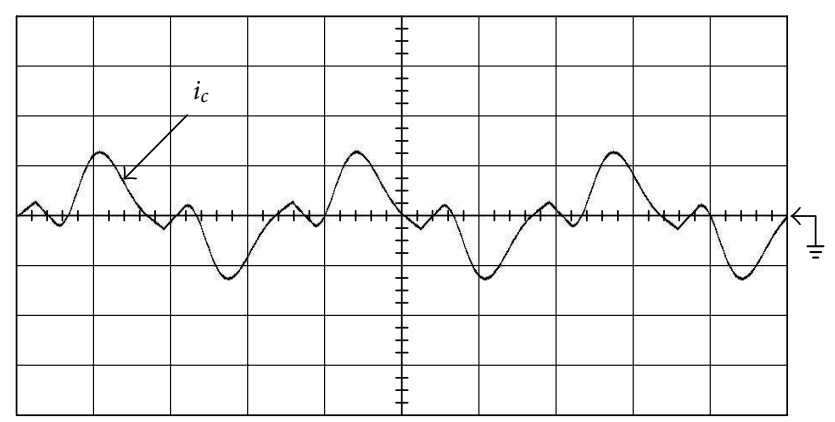

$\left(i_{c}: 5 \mathrm{~A} / \mathrm{div}\right.$, time: $\left.5 \mathrm{~ms} / \mathrm{div}\right)$

FIGURE 19: The corresponding inverter current during the interval of medium insolation.

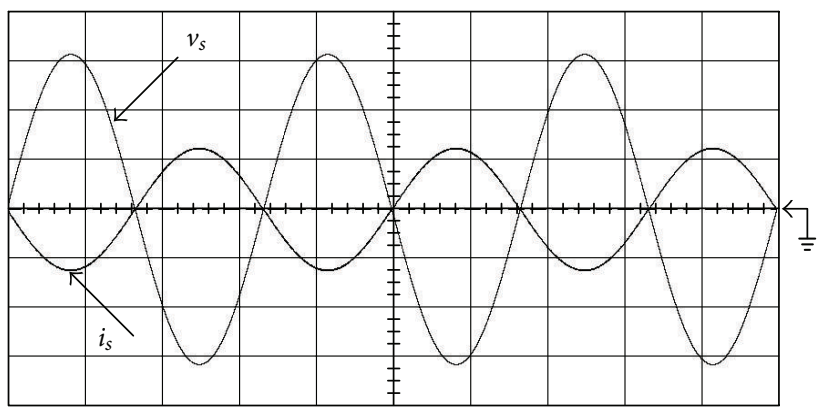

$\left(v_{s}: 50 \mathrm{~V} / \mathrm{div}, i_{s}: 10 \mathrm{~A} / \mathrm{div}\right.$, time: $\left.5 \mathrm{~ms} / \mathrm{div}\right)$

Figure 20: Filtered line current and line voltage during the interval of low insolation.

there is no insolation, the reference voltage is $395 \mathrm{~V}$ and the half-bridge inverter provides reactive power and distortion power for nonlinear loads and draws real power from utility for dc loads. The line current and inverter current are shown in Figures 22 and 23, in turn. Figures 24 and 25 present the practical measurements of line currents during the intervals of high insolation and medium insolation, respectively. In the case of no insolation, the filtered line current and line voltage are shown in Figure 26. During the interval of no insolation the PV system is totally in charge of active power filtering. The amplitude of the filtered line current is greater than that in medium insolation. That is, the utility provides more active power to the load.

\section{Conclusions}

A half-bridge PV inverter capable of dealing with power flow bidirectionally is presented in this paper. As compared with full-bridge one, the total number of active switches is reduced by half so that the system configuration is simplified and its cost is lowered significantly. The LAM is applied to obtain an optimal reference voltage for the determination of a current command and to achieve MPPT feature, which avoids sophisticated calculation. The DSCS algorithm is

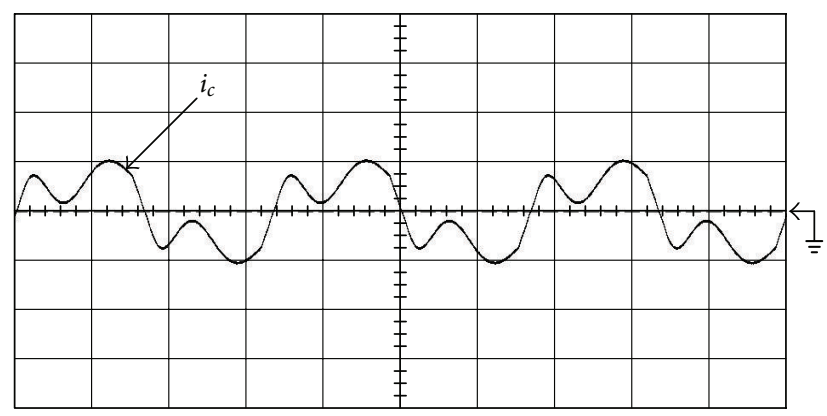

$\left(i_{c}: 5 \mathrm{~A} / \mathrm{div}\right.$, time: $\left.5 \mathrm{~ms} / \mathrm{div}\right)$

FIGURE 21: The corresponding inverter current during the interval of low insolation.

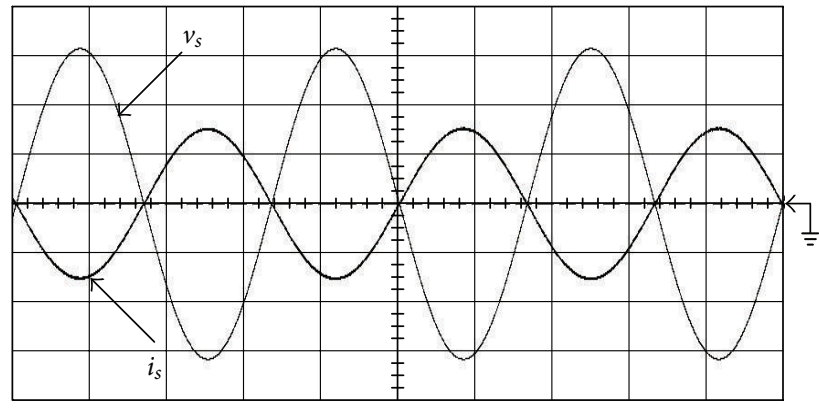

$\left(v_{s}: 50 \mathrm{~V} / \mathrm{div}, i_{s}: 10 \mathrm{~A} / \mathrm{div}\right.$, time: $\left.5 \mathrm{~ms} / \mathrm{div}\right)$

FIGURE 22: Filtered line current and line voltage during the interval of no insolation.

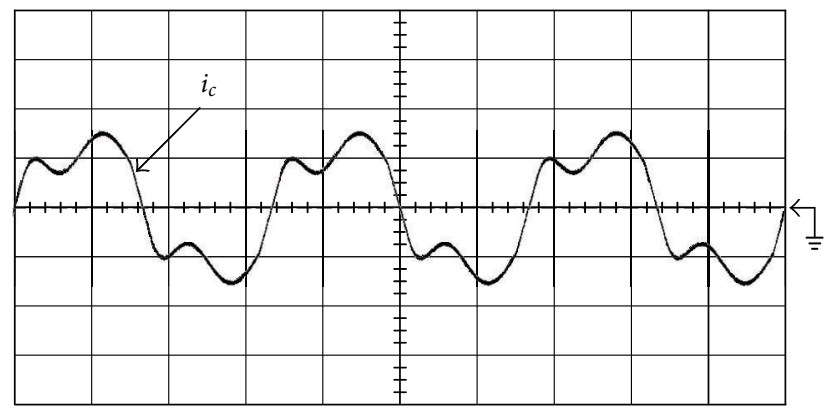

$\left(i_{c}: 5 \mathrm{~A} / \mathrm{div}\right.$, time: $\left.5 \mathrm{~ms} / \mathrm{div}\right)$

FIGURE 23: The corresponding inverter current during the interval of no insolation.

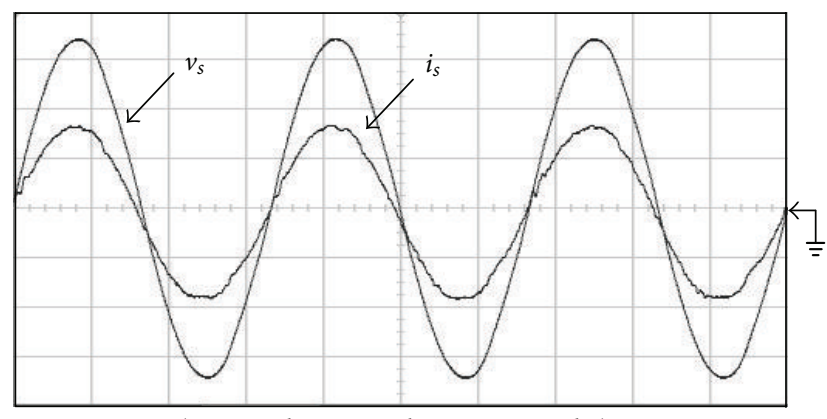

$\left(v_{s}: 50 \mathrm{~V} / \mathrm{div}, i_{s}: 5 \mathrm{~A} / \mathrm{div}\right.$, time: $\left.5 \mathrm{~ms} / \mathrm{div}\right)$

FIGURE 24: Experimental result: the filtered line current and line voltage during the interval of high insolation. 


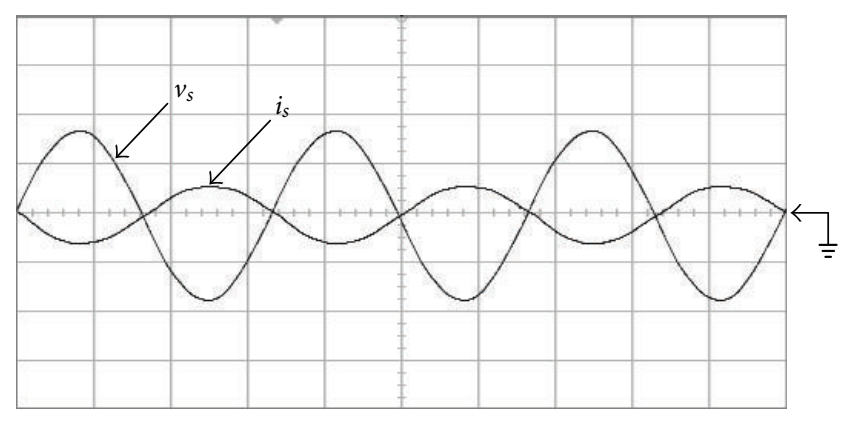

$\left(v_{s}: 100 \mathrm{~V} / \mathrm{div}, i_{s}: 10 \mathrm{~A} / \operatorname{div}\right.$, time: $\left.5 \mathrm{~ms} / \mathrm{div}\right)$

FIgURE 25: Experimental result: the filtered line current and line voltage during the interval of medium insolation.

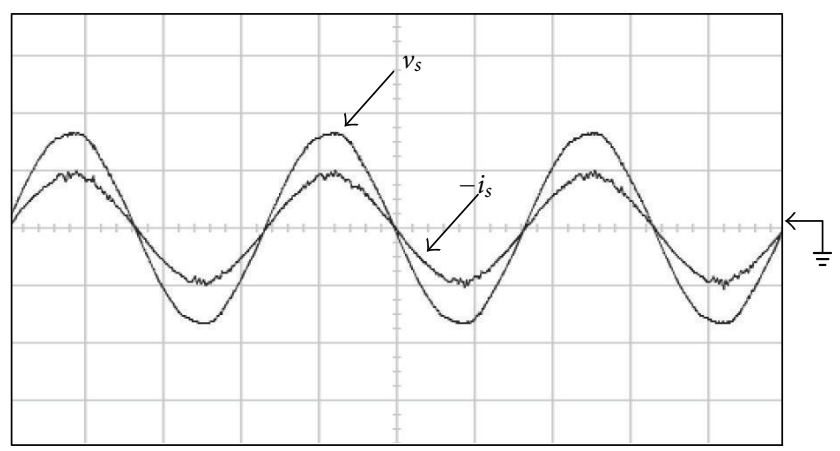

$\left(v_{s}: 100 \mathrm{~V} / \mathrm{div}, i_{s}: 10 \mathrm{~A} / \mathrm{div}\right.$, time: $\left.5 \mathrm{~ms} / \mathrm{div}\right)$

FIGURE 26: Experimental result: the line voltage and the filtered line current during the interval of no insolation.

embedded to perform wave shaping for line current so as to achieve power quality improvement directly. Simulation results and practical measurements have demonstrated the feasibility of the PV inverter system.

\section{References}

[1] L. Asiminoaei, R. Teodorescu, F. Blaabjerg, and U. Borup, "A digital controlled PV-inverter with grid impedance estimation for ENS detection," IEEE Transactions on Power Electronics, vol. 20, no. 6, pp. 1480-1490, 2005.

[2] T.-F. Wu, C.-L. Shen, C.-H. Chang, and J. Chiu, " $1 \phi 3$ W gridconnection PV power inverter with partial active power filter," IEEE Transactions on Aerospace and Electronic Systems, vol. 39, no. 2, pp. 635-646, 2003.

[3] S. A. Daniel and N. AmmasaiGounden, "A novel hybrid isolated generating system based on PV fed inverter-assisted wind-driven induction generators," IEEE Transactions on Energy Conversion, vol. 19, no. 2, pp. 416-422, 2004.

[4] H. Koizumi, T. Mizuno, T. Kaito et al., "A novel microcontroller for grid-connected photovoltaic systems," IEEE Transactions on Industrial Electronics, vol. 53, no. 6, pp. 1889-1897, 2006.

[5] T.-F. Wu, H.-S. Nien, C.-L. Shen, and T.-M. Chen, "A singlephase inverter system for PV power injection and active power filtering with nonlinear inductor consideration," IEEE Transactions on Industry Applications, vol. 41, no. 4, pp. 1075-1083, 2005.

[6] P. P. Barker and J. M. Bing, "Advances in solar photovoltaic technology: an applications perspective," in Proceedings of the IEEE Power Engineering Society General Meeting, pp. 19551960, June 2005.

[7] B. M. T. Ho and H. S. H. Chung, "An integrated inverter with maximum power tracking for grid-connected PV systems," IEEE Transactions on Power Electronics, vol. 20, no. 4, pp. 953962, 2005.

[8] P. G. Barbosa, H. A. C. Braga, M. C. B. Rodrigues, and E. C. Teixeira, "Boost current multilevel inverter and Its application on single-phase grid-connected photovoltaic systems," IEEE Transactions on Power Electronics, vol. 21, no. 4, pp. 11161124, 2006.

[9] J. J. Negroni, C. Meza, D. Biel, and F. Guinjoan, "Control of a Buck inverter for grid-connected PV systems: a digital and sliding mode control approach," in Proceedings of the IEEE International Symposium on Industrial Electronics (ISIE '05), pp. 739-744, June 2005.

[10] C. Rodriguez and G. A. J. Amaratunga, "Dynamic maximum power injection control of AC photovoltaic modules using current-mode control," IEE Proceedings of Electric Power Applications, vol. 153, no. 1, pp. 83-87, 2006.

[11] A. Kotsopoulos, P. J. M. Heskes, and M. J. Jansen, "Zerocrossing distortion in grid-connected PV inverters," IEEE Transactions on Industrial Electronics, vol. 52, no. 2, pp. 558565, 2005.

[12] N. Kasa, T. Iida, and L. Chen, "Flyback inverter controlled by sensorless current MPPT for photovoltaic power system," IEEE Transactions on Industrial Electronics, vol. 52, no. 4, pp. 11451152, 2005.

[13] N. Femia, D. Granozio, G. Petrone, G. Spagnuolo, and M. Vitelli, "Optimized one-cycle control in photovoltaic grid connected applications," IEEE Transactions on Aerospace and Electronic Systems, vol. 42, no. 3, pp. 954-971, 2006.

[14] A. O. Zué and A. Chandra, "Simulation and stability analysis of a $100 \mathrm{~kW}$ grid connected LCL photovoltaic inverter for industry," in Proceedings of the IEEE Power Engineering Society General Meeting (PES '06), June 2006.

[15] T.-F. Wu, C.-L. Shen, H.-S. Nein, and G.-F. Li, "A $1 \phi 3 \mathrm{~W}$ inverter with grid connection and active power filtering based on nonlinear programming and fast-zero-phase detection algorithm," IEEE Transactions on Power Electronics, vol. 20, no. 1, pp. 218-226, 2005.

[16] Y. Chen and K. M. Smedley, "A cost-effective single-stage inverter with maximum power point tracking," IEEE Transactions on Power Electronics, vol. 19, no. 5, pp. 1289-1294, 2004.

[17] K. S. Phani Kiranmai and M. Veerachary, "A single-stage power conversion system for the PV MPPT application," in Proceedings of the IEEE International Conference on Industrial Technology (ICIT '06), pp. 2125-2130, December 2006.

[18] R. González, J. López, P. Sanchis, and L. Marroyo, "Transformerless inverter for single-phase photovoltaic systems," IEEE Transactions on Power Electronics, vol. 22, no. 2, pp. 693697, 2007. 


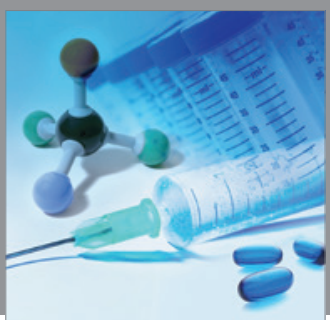

International Journal of

Medicinal Chemistry

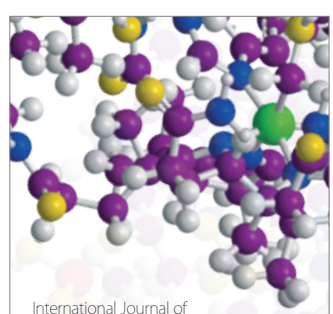

Carbohydrate Chemistry

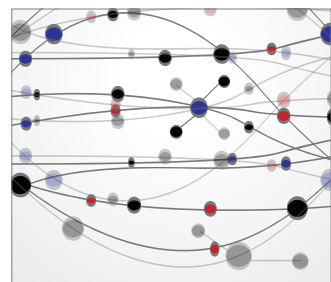

The Scientific World Journal
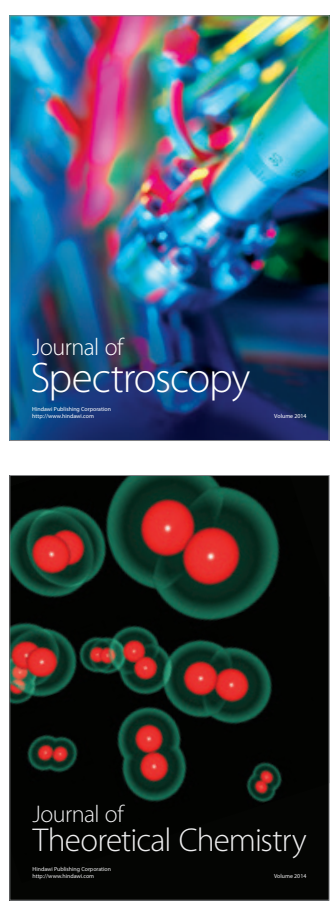
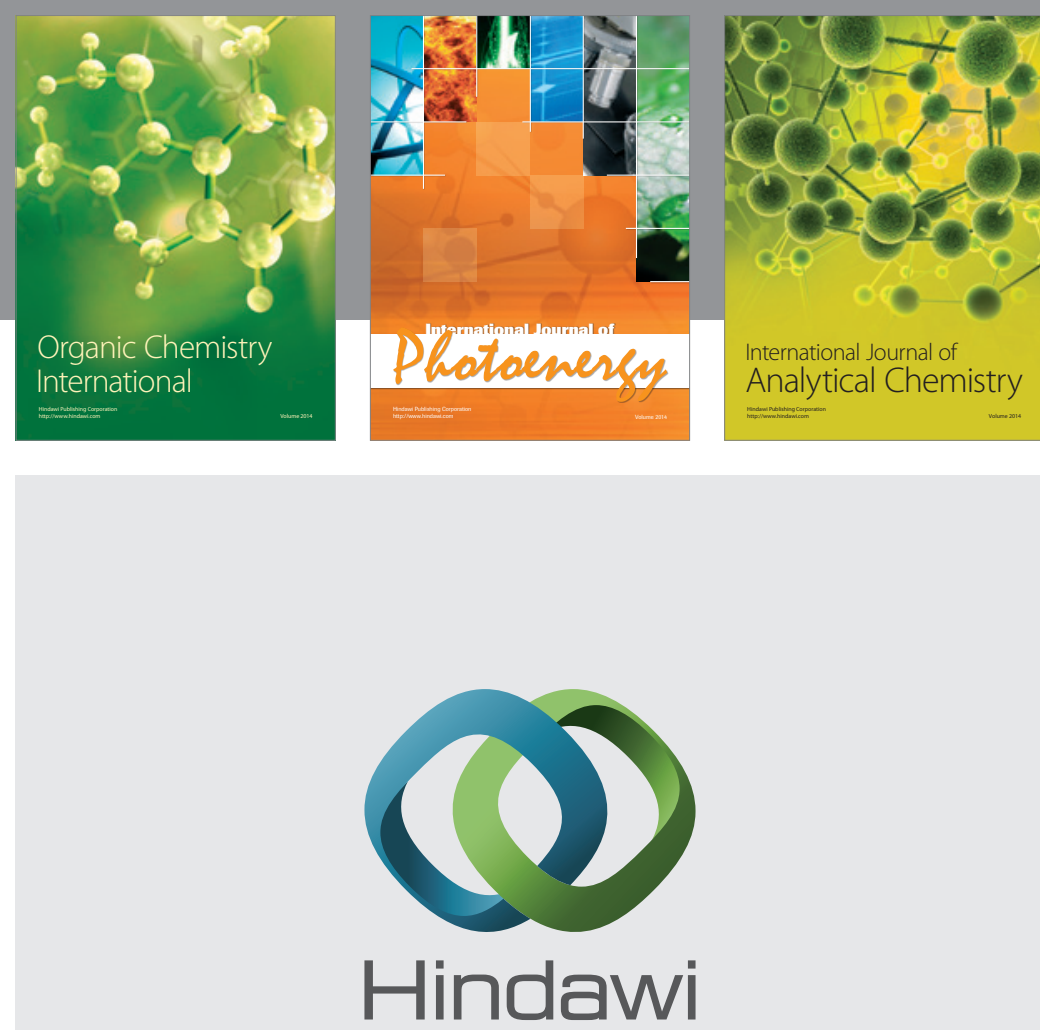

Submit your manuscripts at

http://www.hindawi.com
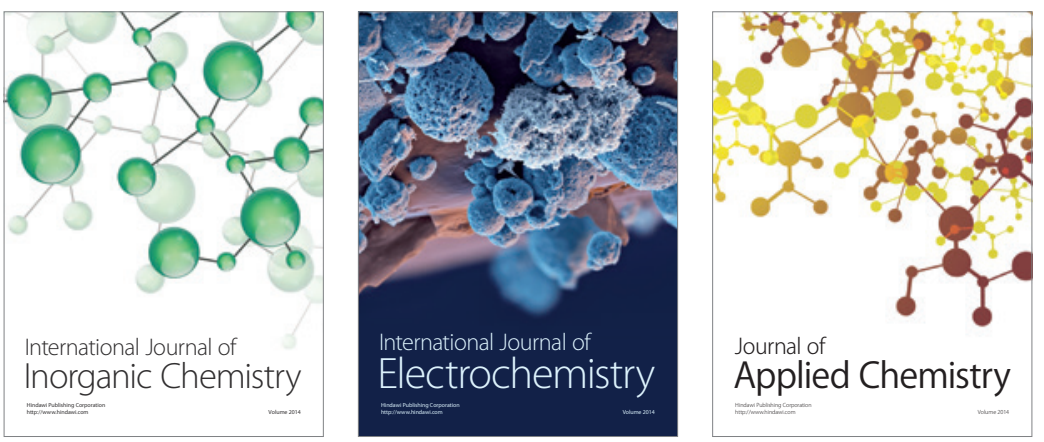

Journal of

Applied Chemistry
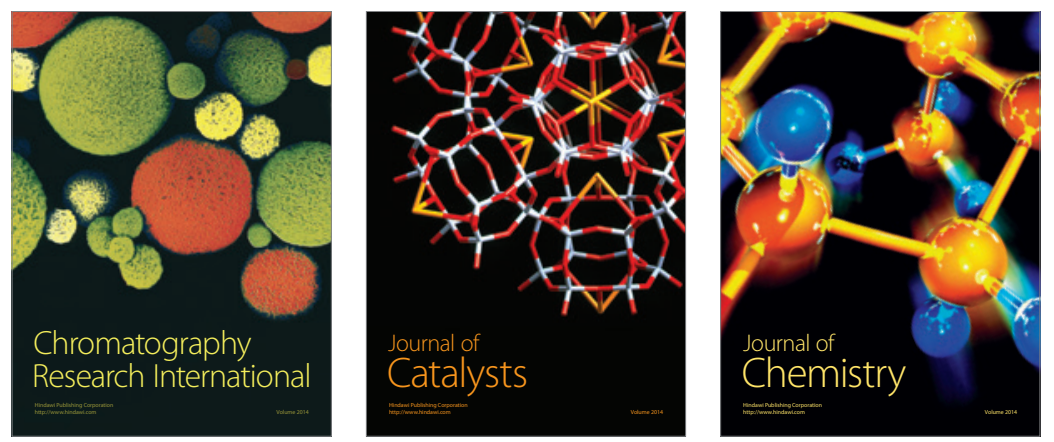
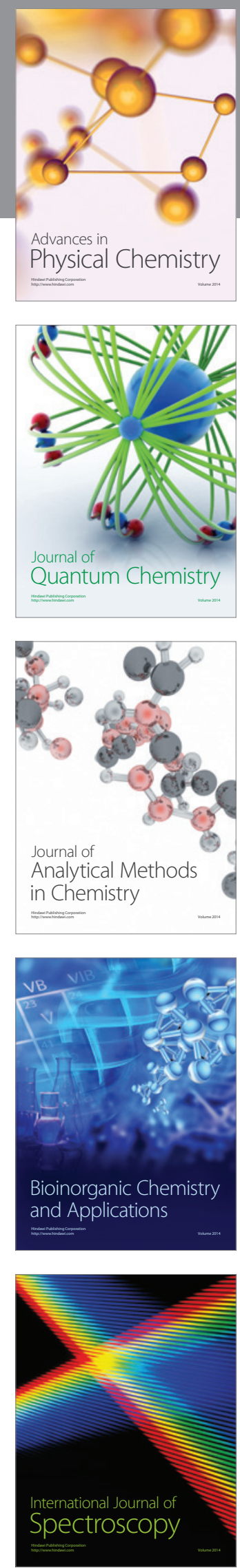